\title{
PROSPECTS
}

\section{Search tool for career data launched}

\section{A new online facility allows users to delve into Naturejobs's career and salary survey data on their own terms, explains Gene Russo.}

In late June, we released an analysis of many of the results from Nature and Naturejobs's first international salary survey, which received more than 10,500 responses from scientists worldwide (see go.nature.com/ $\mathrm{R} \vee v q W e$ ). It detailed trends related not only to scientists' incomes, but also to their job satisfaction. Accompanying the analysis were expert commentaries (see go.nature. com/8Z6tnr) and a podcast sampling some of the survey's more interesting outcomes (see go.nature.com/VwUzzp).

This week, we are offering readers an additional online-only resource, free with Naturejobs registration. Our search tool will allow users to probe the data set in even more detail - on their own terms and according to their own interests (see go.nature.com/xV3dnZ).

Using the tool is intuitive. Readers first select a topic from the drop-down menu, such as salary, then a filter - such as career stage or satisfaction. The results are instantly available as a graph and a table.

Interested in how much money our biologist respondents make? Choose 'Current salary of primary job', then filter with 'Primary subject/research area' and 'Biology'. The large number of scientists on US\$40,000-50,000 reflects in part the large number of postdoc respondents (which, in turn, reflects the large number of postdocs out there).

Want to know how biologists rate the different sources of job satisfaction? Choose 'Sources of job satisfaction' and filter with 'Primary subject/research area' and 'Biology'. Users can generate quite subtle results. Try choosing 'Sources of job satisfaction', then filtering 'Overall job satisfaction' with the subcategory 'Very satisfied'. The results suggest that among those who reported being very satisfied with their current position, 'degree of independence' and 'guidance received from co-workers' were among the leading factors contributing to contentment.

We have chosen to display and make available for selection only categories and subcategories with sample sizes of at least 50 . This means that data from many countries those for which we had small sample sizes will not be available. And, as explained in our frequently-asked-questions section, there are biases in the data that we have done our best to address (see go.nature.com/MP91Ge).

We hope this new tool helps inform, enlighten and even entertain, whether users are browsing the results, using them for a presentation or simply sharing them with colleagues.

Gene Russo is the editor of Naturejobs.

Stefania Mondello, a postdoc at the University of Florida, received the 2010 Premio Award, presented to outstanding female Italian scientists working in North America, at the annual conference of the Italian Association for Women Inventors and Innovators in Bari, Italy, in June.

What attracted you to biology? As a child I read about Louis Pasteur, creator of the first vaccine for rabies, and Alexander Fleming, discoverer of penicillin. Their research contributed to a better world and I decided I wanted to be a physician-scientist to do the same.

\section{Why did you focus on} traumatic brain injury? While at the University of Messina Medical School in Italy, I began to focus my PhD training on neural intensive care. I found it fascinating that you need to know how so many organs are related to treat these patients, the brain being the most complex. I did my thesis on how best to use biomarkers - molecules that can serve as indicators of disease progression - as targets for therapy. Yet none existed for traumatic brain injury, so I decided to focus on developing these biomarkers. People with traumatic brain injuries are often young people hurt in dramatic situations. Their recovery depends on accurate, early treatment that takes into account the person's genetics.

How is your awardwinning work innovative?

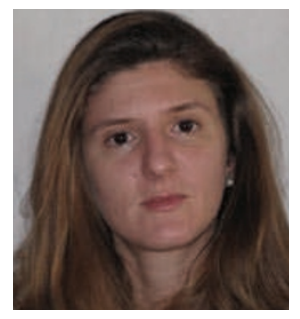

got it without the strong team effort needed to do this type of research. My mentors helped me develop a unique set of skills - merging my clinical focus on the brain with a We aim to use biomarkers to knowledge of biomarkers and assess the magnitude of the brain injury as well as to help determine the most effective treatment. People can seem to have a mild-to-moderate brain injury, yet it is impossible to accurately measure the damage. We hope our work will one day change medical practice to prevent secondary complications and improve the survival of the 1.4 million people in the United States alone who experience a traumatic brain injury each year.

You're only 31 - were you surprised to get the award? Yes, I was. I left Italy only two years ago, and these types of award are rarely given to someone so young. But I would not have genetics - and this award just makes me want to work harder to use those skills to find treatments.

\section{What are your long-term} goals?

Now that we've found diagnostic biomarkers, we are working on their clinical validation - confirming the different biomarkers in people with traumatic brain injury compared with controls, assessing their diagnostic accuracy, and demonstrating that they provide information that aids medical decision-making. Once we have clinical validation, our goal is to secure US Food and Drug Administration approval by 2012 . The agency has not approved a new treatment for traumatic brain injury in the past 30 years.

What opportunities come with this award?

The award is given by Bridges to Italy, which supports women scientists and fosters collaborations between Italy and the United States. As biomedicine in Italy continues to move towards conducting science with more clinical utility, I hope to advocate for more investment in young investigators.

\section{What inspires you?}

Two quotes illustrate my belief that practical people are important, but so are dreamers. My mentor, Ronald Hayes, said: "We seek the truth as scientists, we heal the sick as clinicians, and we teach others to do the same as mentors." And Marie Curie said: "A scientist is not only a technician; he is also a child placed before natural phenomena which impress him like a fairy tale." Interview by Virginia Gewin. 